\title{
REVISIÓN DE PARES EN UN CURSO DE ESCRITURA CREATIVA EN INGLÉS: FOCO Y POSICIONAMIENTO DE LOS REVISORES
}

\section{PEER REVIEW IN A CREATIVE WRITING COURSE: FOCUS AND REVIEWERS' POSITIONING}

\section{Sol Colmenares Rodríguez ${ }^{1}$}

\author{
Alexánder Ramírez Espinosa² \\ Universidad del Valle, Cali - Colombia
}

\section{RESUMEN}

\section{PALABRAS CLAVE}

Este artículo reporta los resultados de un estudio de caso de corte descriptivo-interpretativo en el curso de Escritura Creativa en Inglés, que hace parte de un programa de Licenciatura en Lenguas Extranjeras. El objetivo principal fue describir las revisiones hechas por estudiantes y establecer, por un lado, en qué aspectos del texto se enfocan, y por otro cómo se posicionan los estudiantes en su rol de revisores. El corpus, constituido por 46 revisiones escritas por 25 estudiantes durante el primer semestre de 2020, fue sometido a un análisis temático. Los resultados sugieren que, en este caso, las revisiones trascendieron el foco tradicional de la corrección lingüística y se centraron en aspectos globales y estilísticos del texto tales como la construcción de personajes y la construcción de audiencia.

\footnotetext{
1 Universidad del Valle, Cali - Colombia sol.colmenares@correounivalle.edu.co

$2 \quad$ Universidad del Valle, Cali - Colombia alexander.ramirez.e@correounivalle.edu.co https://orcid. org/ 0000-0002-7122-9537
}

Revisión de Pares, Escritura Creativa, Enfoque Basado en Tareas, Posicionamiento

\section{ABSTRACT}

This article reports the results of a descriptiveinterpretive case study in a Creative Writing course, from a Foreign Language Teacher Education program. The main objective was to describe the reviews made by students and establish, on the one hand, the aspects of the text they focused on, and on the other, how students position themselves in their role as reviewers. A corpus of 46 reviews written by 25 students during the first semester of 2020 was collected, and subjected to thematic analysis. The results suggest that, in this case, the reviews transcended the traditional focus of linguistic correction and focused on global and stylistic aspects of the text such as character construction and audience construction. 


\section{KEYWORDS}

Peer Review, Creative Writing, Task Based Approach, Positioning

\section{INTRODUCCIÓN}

La revisión por pares, una práctica editorial característica de las revistas científicas, tiene como principal objetivo mejorar la calidad de los artículos sometidos a evaluación a partir de la crítica constructiva que otros investigadores ofrecen al autor. Considerada un importante instrumento de avance de la ciencia, la revisión es "una defensa del lector" y "un ejercicio educativo para el autor y el evaluador" (Gérvas \& Pérez, 2001, p. 433), razones que seguramente han contribuido a su adopción como estrategia pedagógica por docentes de escritura en distintos niveles y disciplinas (López, 2012; Vargas, 2014; Chois et al., 2017; Salinas, 2020; Colombo \& Álvarez, 2021).

Autores como Anson y Anson (2017) reconocen en la evaluación por pares una herramienta que "refuerza la metacognición y que tiene efecto tanto en el mejoramiento inmediato de un texto en desarrollo como en la transferencia potencial de perspectivas y habilidades retóricas y lingüísticas a otros contextos" (p. 21, traducción de los autores ${ }^{3}$ ). Paton (2002), por su parte, le atribuye la facultad de promover el desarrollo de habilidades interpersonales tales como: "la habilidad para escuchar respetuosamente, dar voz a las opiniones abiertamente, aconsejar con delicadeza y negociar puntos de vista diferentes" (p. 291). En la misma línea de nombrar las bondades de esta práctica pedagógica, Sanabria (2015) señala que en la edición por pares "el salón de clase se convierte en un escenario social donde los estudiantes interactúan con el propósito de apoyarse y aprender juntos" (p. 86).

3 En adelante, todas las citas tomadas de textos escritos originalmente en inglés corresponden a la propuesta de traducción de los autores.
Uno de los supuestos más interesantes que subyacen a la revisión de pares es, a nuestro juicio, la visión del texto como "contingente y abierto a la respuesta y la crítica", una perspectiva que se inscribe dentro de una "comprensión social y constructivista del trabajo académico" (Schneider \& Andre, 2007, p. 9) y que exige el uso de "tiempo de clase para desarrollar habilidades grupales y para aprender a ofrecer y recibir retroalimentación" (Harris, 1992, p. 381). En este marco, evaluar la escritura de otros se convierte en una tarea conjunta en la que "un grupo de personas busca valores y significados a través de la interpretación grupal". (Huot, 2002, p.173). De ahí que, para que la revisión de pares resulte una experiencia pedagógica enriquecedora, es necesario un "auténtico sentido de comunidad dentro de la clase como un todo" (Paton, 2002, p. 295). Cuando se logra ese sentido, se comparte lo que se escribe en un ambiente de seguridad y confianza y se lee al otro con responsabilidad.

Sobre ese trasfondo, el propósito de este artículo es el de compartir una experiencia de revisión por pares en un curso de escritura creativa en inglés. Este curso forma parte del currículo de un programa de Licenciatura en Lenguas Extranjeras (Inglés-Francés), en una universidad pública colombiana. Se trata de un curso electivo que se ofrece a estudiantes de séptimo semestre en adelante, es decir que los estudiantes que allí se inscriben han alcanzado un nivel intermedio-avanzado de proficiencia en las lenguas extranjeras que estudian. El curso se desarrolló desde un enfoque basado en tareas (tasks).

\section{LOS ESTUDIANTES COMO PARES EVALUADORES}

Encargar a otro la tarea de revisar un texto entraña un reconocimiento de autoridad basada en la experticia. ¿Qué sucede cuando ese otro no es el profesor sino un compañero tan o más 
neófito o inexperto que yo? El problema más evidente en la revisión -concluye López (2012)-

es la actitud de los estudiantes frente al proceso [...] algunos se sintieron incómodos con el ejercicio $y$, en ocasiones, consideraban que no tenían las suficientes competencias lingüísticas para hacer sugerencias a sus compañeros, a pesar de tener una guía para orientar los comentarios y de haber trabajado los diferentes aspectos tanto en la clase como en sus propios textos (p. 378).

Esta conclusión resuena con uno de los hallazgos de Vargas (2014) quien identifica el caso de estudiantes que "preferían buscar la revisión del profesor (...) después de ser revisados por un par porque consideraban que las correcciones de sus compañeros eran demasiado superficiales (puntuación, ortografía,
Como explicación para esta reticencia al uso de la revisión de pares, consideramos que las preferencias de los estudiantes y sus creencias sobre el valor de la retroalimentación del maestro y de los compañeros pueden incidir en el uso o no de los comentarios recibidos. Las entrevistas de Nelson y Carson (1998) con estudiantes universitarios de L2 revelan que los estudiantes preferían la retroalimentación de los maestros y era mucho más probable que la incorporaran en sus revisiones. En el estudio de Zhang (1995) con 81 estudiantes universitarios de primer año, el $75 \%$ prefirió la retroalimentación del maestro a todas las demás formas. Los estudiantes tienden a confiar en sus profesores más que en sus compañeros, considerando al profesor como el experto y a sus compañeros como agentes que pueden no tener el conocimiento suficiente para diagnosticar sus falencias (Sengupta 1998). De manera similar, Tsui y Ng (2000) encontraron que los estudiantes de secundaria de Hong Kong veían al maestro como una "figura de autoridad que garantizaba la calidad" (p. 149) y se mostraban reacios a confiar en las correcciones de sus compañeros. (Hyland \& Hyland, 2006, p. 91).

Según Patón, el énfasis en aspectos propios de la corrección de estilo tiene lugar, especialmente entre estudiantes de primeros niveles, porque "es el tipo de retroalimentación más obvia y menos arriesgada" y es por ello que resulta necesario "enfatizar la diferencia entre revisión, edición y corrección de estilo y centrar a los estudiantes en la revisión de aspectos sustantivos tales como el enfoque, la audiencia, el desarrollo y la coherencia" (Paton, 2002, p. 292). Huot, por su parte, nos advierte de la responsabilidad que tenemos como profesores cuando señala que "nuestros estudiantes cargan con muchas de las nociones negativas, críticas y centradas en la corrección que tanto prevalecen en la sociedad y entre nosotros, sus profesores de escritura" (Huot, 2002, p. 170). Por otro lado, Vargas (2014) coincide en que los docentes también fallamos cuando pedimos a los estudiantes que "escriban sus trabajos fuera del aula y [suponemos] que estos revisan como escritores expertos cuando no lo son" (Vargas, p. 27).

Estas dificultades se encuentran, de manera quizás más evidente, en contextos de aprendizaje de lenguas extranjeras, en los cuales "los estudiantes tienen problemas para detectar errores y proveer retroalimentación de calidad, recurriendo a veces a comentarios formulaicos, siendo super-críticos o enfocándose excesivamente en errores superficiales" (Hyland \& Hyland, 2006, p. 91). Por esta razón, resulta de suma importancia discutir explícitamente el rol de los estudiantes como revisores para comprender que "no se trata de asumir la posición del docente sino de responder como pares interesados y solidarios, que, aunque no son escritores expertos [...] sí tienen la 
capacidad de ofrecer una crítica constructiva" (Paton, 2002, p. 292).

Este nuevo rol del estudiante como revisor ha de discutirse al interior de un proceso explícito de formación que permita a los aprendices explorar y consolidar diferentes tipos de posicionamiento. Teniendo en cuenta los postulados de Schneider \& Andre (2007), la revisión por pares en el aula de clases incentiva una meta-conversación entre estudiantes/autores que les permite, por un lado, explorar sus experiencias pasadas como lectores, y por otro lado insertarse en dinámicas y discursos propios de su quehacer profesional. Ambos aspectos coadyuvan a la consolidación de un posicionamiento de autoridad que es esencial en la formación profesional, pues aquellos estudiantes que no logran desarrollar un sentido de autoridad "podrían quedar atrapados en los roles de procesador de textos o de legos, subordinados al conocimiento de otros y reacios a adoptar la identidad de legítimos miembros -aunque novatos- de sus disciplinas y profesiones" (Schneider \& Andre, 2007, p.19).

De manera similar, Clark e Ivanic (1997) afirman que tal sentido de autoridad se desarrolla cuando quien escribe es capaz de verse a sí mismo como miembro de un grupo particular, con unas convenciones particulares, y en el que su escritura -y por supuesto su crítica a la escritura de otros- revela su yo autobiográfico (esto es, sus experiencias, sistemas de valores y creencias), su yo discursivo (sus características retóricas y estilísticas), y su yo autoral (su posicionamiento como autor y como autoridad). En otras palabras, la formación de estudiantes en su rol de pares evaluadores debe permitirles desarrollar la confianza para posicionarse como revisores legítimos que pueden contribuir al proceso de escritura de otros a partir de sus propias experiencias como lectores y escritores, así como desarrollar consciencia de que ellos mismos, en tanto autores, verán siempre expuesta su producción escrita al escrutinio de otros.

\section{REVISIÓN DE PARES Y ESCRITURA CREATIVA EN INGLÉS COMO LENGUA EXTRANJERA}

El uso de la revisión de pares como estrategia pedagógica en contextos de enseñanzaaprendizaje de escritura en inglés como lengua extranjera no es "un concepto novedoso" (Coté, 2014, p.69). Al contrario, "especialmente popular en la enseñanza de lenguas extranjeras (...) ha demostrado ayudar a los estudiantes a entender su propio proceso de desarrollo en la escritura a través del análisis de la escritura de pares que se encuentran en etapas similares" (Anson \& Anson, 2017, p.14). Este auge ha dado lugar a "investigaciones sobre los roles de la revisión de pares en la enseñanza de escritura. Sin embargo, las dinámicas de la interacción y las estrategias que median la comprensión de la retroalimentación por parte de los escritores no han sido exploradas de manera suficiente" (Zhao, 2018, p. 263).

Como se señaló en un apartado anterior, las dificultades que se encuentran al usar la revisión de pares parecen aún más evidentes en contextos de aprendizaje de lengua extranjera ya que es posible que los estudiantes no hayan desarrollado "las habilidades comunicativas y pragmáticas necesarias para una interacción exitosa" (Hyland \& Hyland, 2006, p. 92). No obstante, esta carencia puede convertirse en una oportunidad de aprendizaje ya que "estas lagunas o fallas en la comunicación impulsan a los interlocutores a utilizar diferentes estrategias creando así espacios para aprender la segunda lengua" (Gass y Mackey, 2005 en Rodas \& Colombo, 2021, p. 2). Los "beneficios cognitivos, afectivos, sociales y lingüísticos de la revisión de pares" tanto en lengua materna como en lengua extranjera "han sido corroborados" según Min 
(2006) "con evidencia empírica existente" (Min, 2006, p. 118).

En los talleres de escritura creativa, por otra parte, la revisión de pares es una práctica muy común. Akhtar (2015) la considera el "fundamento de la mayoría de talleres" (Akhtar, 2015 , p. 1), aunque no por eso sea una tarea fácil; Norton (2013), por ejemplo, llama la atención sobre las dificultades que representa "medir" o arbitrar una producción tan compleja como la escritura creativa, sobre todo porque sopesar la calidad del texto depende del gusto estético personal del lector/revisor: "la tontería en un lector es poesía para otro" (Norton, 2013, p.5). Tal vez por eso se suelen separar las prácticas de escritura académica y de escritura creativa, en una disparidad que Norton (2013) considera desmoralizante: "las instituciones académicas han establecido oposiciones tácitas binarias entre 'lo académico y lo creativo', 'lo intelectual y lo artístico,' oposiciones que no son mutuamente excluyentes en lo absoluto" (Norton, 2013, p.5). En esa misma línea, y más particularmente en el contexto de enseñanza de lenguas extranjeras, Smith (2013) advierte sobre una paradoja de los textos en la que, por un lado, la producción creativa es altamente valorada y se recomienda la lectura de obras literarias, así como el consumo de otras formas artísticas como películas y música; pero, por otro lado, en el aula se les pide a los estudiantes que produzcan principalmente textos académicos.

No obstante, y pese a los retos que implica corregir la producción artística de otro, la revisión de pares en escritura creativa, particularmente en el contexto de enseñanza de lenguas extranjeras, resulta altamente provechosa, sobre todo si tenemos en cuenta que explorar la producción creativa/literaria potencializa diversos aspectos de la adquisición de segundas lenguas tales como vocabulario, estructuras gramaticales, competencias pragmáticas, construcción del humor, juegos lingüísticos, además de motivación hacia el aprendizaje de la lengua extranjera y desarrollo de la voz autoral en L2 (Smith 2013, Carter, 2004; Belz $\&$ Reinhart, 2004; Cook, 2000). En este sentido, la revisión de textos por pares en el aula de lenguas extranjeras constituye una herramienta valiosa para exponer a los estudiantes a los repertorios lingüísticos, culturales y estilísticos de sus compañeros.

\section{EL ENFOQUE BASADO EN TAREAS Y LA ESCRITURA CREATIVA}

Como se ha dicho anteriormente, el curso se basó en un enfoque basado en tareas (TaskBased Approach). Este enfoque permite que los aprendices de lenguas desarrollen habilidades lingüísticas mientras centran su atención en la resolución de tareas o problemas comunicativos. En el caso de Creative Writing, más que la lengua en sí, nuestro propósito de enseñanza a través de las tareas fue el proceso de escritura, así como la exploración y desarrollo de la creatividad personal en las producciones escritas. Al respecto, Madashi y Dadari (2012) comentan que el enfoque basado en tareas favorece la capacidad creativa y el pensamiento crítico, a la vez que fomenta el desarrollo de habilidades lingüísticas de manera paralela. Hay cuatro características de las tareas, adaptadas de Ellis (2009) y Hout (2002), que fundamentan nuestra escogencia de este enfoque como un aliado para la enseñanza de la escritura creativa. Primero, la estructura tripartita de las tareas (pre, durante y post) va de la mano con el proceso de escritura: este es un proceso de revisión, de ir y venir, de hacer un seguimiento del progreso de su escritura. La pre-tarea equipa a los estudiantes con las herramientas $\mathrm{e}$ insumos necesarios para afrontar el desafío de la escritura; la tarea en sí constituye el momento creativo del estudiante; finalmente la posttarea es el momento que da paso a la revisión por pares, a la corrección y edición, e incluso 
a la articulación del texto a otras producciones previas del mismo autor.

La segunda característica de las tareas es su adaptabilidad. Las tareas se pueden adaptar en términos de nivel de idioma y edad de los participantes. Esto significa que una vez que se diseña una tarea, se puede utilizar con un grupo de estudiantes principiantes o avanzados, ya que cada uno propondrá una solución al problema en la medida de sus habilidades lingüísticas. Asimismo, partimos del hecho de que la mayoría de ejercicios de escritura que se plantean a través de una tarea se pueden proponer a niños, adolescentes o adultos, ya que cada grupo de edad tendrá un punto de vista válido de la realidad, que será susceptible de ser plasmado con una estética particular en la producción textual.

La tercera característica que hace de las tareas un enfoque aliado en la enseñanza de escritura creativa es la relevancia y conexión con la realidad. Las tareas proponen situaciones comunicativas reales que requieren como respuesta una actuación lingüística real. En este sentido, las tareas no emulan, por ejemplo, un diálogo en el aeropuerto, sino que propondrán una llamada telefónica real a una agencia de viajes para recuperar información. En la escritura creativa, esta característica resulta ser un gran detonador de motivación para los estudiantes de idiomas, ya que las tareas propuestas implican la escritura de un texto que supone un objetivo claro, una audiencia real que no se reduce al docente y, por lo tanto, una producción original y genuina.

Finalmente, las tareas redundan en una colección de textos que permite la elaboración de portafolios de escritura. Estos portafolios constituyen un registro del proceso editorial y autoral de los estudiantes en el que estos pueden rastrear la evolución de sus habilidades, la consecución de su voz y estilo propios, y el despliegue de su creatividad. Esa mirada retrospectiva a la producción escrita es también parte de la constante evaluación y autoevaluación, tan importantes en el proceso editorial. Haciendo referencia a los portafolios, Hout (2002) comenta que "el acto de escribir y la habilidad de hablar sobre dicha escritura promueve un discurso pedagógico que no sólo resalta la producción escrita del estudiante y el proceso que genera tal producción, sino también el desarrollo de dicho estudiante en tanto escritor" (p. 173).

\section{METODOLOGÍA}

\section{Tipo de estudio}

Se llevó a cabo un estudio de caso de corte descriptivo-interpretativo en el curso de Escritura Creativa en Inglés (Creative Writing), que hace parte del programa de Licenciatura en Lenguas Extranjeras (Inglés-Francés) de la Universidad del Valle en Cali, Colombia. El objetivo principal del estudio fue describir las revisiones hechas por estudiantes en términos de foco y posicionamiento de los revisores. Las siguientes preguntas guiaron el estudio:

- ¿Cómo son las revisiones hechas por los estudiantes? ¿En qué aspectos del texto se enfocan?

- ¿Cómo se posicionan los estudiantes en su rol de revisores? ¿Qué estrategias de mediación y qué patrones de interacción usan?

\section{CORPUS, CONTEXTO Y PARTICIPANTES}

El corpus sometido a análisis lo componen 46 revisiones escritas por dos grupos de estudiantes del curso Creative Writing (ofrecido como materia electiva en el programa de Licenciatura) durante el primer semestre de 2020. El curso se ofreció a 25 estudiantes, repartidos en dos 
grupos, todos ellos con un nivel de B2 o superior en inglés, según el Marco Común Europeo para las lenguas. Todos los estudiantes participaron en el estudio de manera voluntaria y autorizaron a los docentes el uso de sus producciones, así como el de las revisiones hechas por sus pares con fines investigativos. A lo largo del curso, especialmente durante las primeras clases, se realizaron varias sesiones en las que se discutió el tema de la revisión de pares y su lugar dentro del programa. Dos de los cuatro objetivos del curso se plantearon con relación a esta metodología: (1) Desarrollar habilidades para la revisión de pares de manera significativa, generosa y respetuosa y (2) Exponerse a la mirada del otro y aprender a recibir críticas que ayuden a revisar el propio trabajo.

La distribución de pares para la revisión se hizo de manera aleatoria pero no anónima. Luego, los estudiantes tuvieron la posibilidad de escoger cuál(es) de la(s) tareas de sus compañeros querían retroalimentar. Todos los estudiantes fueron tanto autores como revisores, desempeñando este último rol una o dos veces durante todo el curso. Los textos revisados fueron escritos en respuesta a un compendio de diez tareas de escritura que fueron diseñadas teniendo en cuenta la estructura tripartita mencionada anteriormente, que incluye las etapas Pre (Insumos, ejemplos), Durante (Escritura) y Post (retroalimentación, evaluación de pares, conexión con otras tareas).

\section{TRATAMIENTO Y ANÁLISIS DE LOS DATOS}

Las revisiones por pares se recogieron en portafolios virtuales usando las aplicaciones de Google Drive y un blog de Wordpress. Una vez recolectadas las revisiones, se procedió a hacer un análisis temático (Braun \& Clarke, 2006). Estas autoras definen el análisis temático como un método cualitativo para identificar, organizar, describir, analizar y reportar patrones -o temasencontrados en los datos, y así poder dar una mirada amplia e interpretar los fenómenos de interés del investigador. A diferencia del análisis de contenido, que es un método muy similar y bastante popular en los estudios de textos escritos, el análisis temático desemboca en un reporte puramente cualitativo y detallado (Braun \& Clarke, 2006), mientras que el primero usa un enfoque cuantitativo tanto para codificar los datos, como para la interpretación de las categorías resultantes de la codificación (Vaismoradi et al., 2013, p.13).

Teniendo en cuenta la naturaleza flexible del análisis temático, así como el procedimiento propuesto por Braun y Clarke (2006), se llevaron a cabo las siguientes etapas:

- Aproximación al corpus: Se hizo una lectura sistemática de las revisiones, e incluso se recurrió a la transcripción de algunas de ellas, técnica que se recomienda para familiarizarse con los datos (Vaismoradi, 2013; Braun \& Clarke, 2006).

- Definición de Categorías y Codificación: Se establecieron dos categorías iniciales: Foco, y Posicionamiento de los Revisores. Se dio inicio a una codificación por colores para identificar temas relacionados con estas dos categorías, manteniendo siempre la apertura hacia potenciales categorías y temas emergentes.

- Correlato de Códigos y Definición de Nuevos Temas: Una vez terminada la codificación, se procedió a hacer el correlato de los códigos de colores, darle un nombre a los temas identificados, así como a las categorías que emergieron del análisis.

- Producción del Reporte: finalmente, se hizo una revisión general de todos los 
temas, y se generó el reporte descriptivointerpretativo en el que se proporcionan extractos de las revisiones a manera de ilustración. Cada revisión fue codificada con un número que aparece al final de la cita cada vez que se presenta un extracto. También se reemplazaron los nombres reales de los participantes por seudónimos.

\section{RESULTADOS Y DISCUSIÓN}

A continuación presentamos los resultados del análisis intercalados con ejemplos extraídos del corpus. Los ejemplos incluidos mantienen fielmente la lengua y formato original en la que fueron escritas las revisiones, y se identifican con una "P" de "peer-review" y el número de la revisión de la que se extrajo cada ejemplo.

¿En qué se enfocaron las revisiones por pares?

En las revisiones analizadas se identificaron como objeto de valoración seis categorías, las cuales se corresponden con los siguientes elementos del texto: la construcción de personajes, el cumplimiento de la tarea, el estilo, el tema y la construcción de audiencia. En menor medida, se encontraron algunas referencias a la gramática, la estructura o el léxico; sin embargo, cabe anotar que, en algunos casos, dichas referencias a cuestiones formales de la lengua comportan una intención estilística, es decir que van más allá de la mera corrección idiomática. Un fenómeno frecuente al analizar el corpus fue la superposición de estos temas, es decir que en un mismo extracto es posible encontrar evidencias de aproximación a más de un aspecto del texto.

Con respecto a la construcción de personajes, tal como puede apreciarse en los siguientes ejemplos, los revisores que abordaron este tema valoraron de manera positiva la verosimilitud y la coherencia interna de los mismos:

She personified what I would call a traditional mom: loving her role of being a mom in contrast to loving her child and that's the part when it gets not so pleasant, being ashamed of her boy, making him guilty for socially modelled expectations [...] And that makes such a good work for Sasha, as she crafted this hateful character. I can totally see thousands of these characters all over the city and the world (...) (P14)

I also liked the duality of the character itself, because even if she is $100 \%$ sure she wants a relationship with him, she is constantly telling herself "maybe" or "I don't know" as if she were looking for protecting herself from his possible rejection, even in her mind. (P19)

La posibilidad de identificarse con los sentimientos de los personajes también se evalúa de forma positiva: "The description of the situation and the attention to detail makes you feel what the characters are experiencing" (P09) mientras que la falta de claridad en la construcción de la voz y la necesidad de desarrollar los conflictos y motivaciones internas son señalados como aspectos a mejorar:

At some point in our lives, we all have experienced what it's like to hit Rock bottom, just like Mary seems to be doing, so it's relatable. (P40)

The character of the mother reveals a duality: the precepts of her religion and her unconditional love for her son (...) How strong is that inner conflict 
for her? I think giving a deeper view of the mother's conflict would intensify the conflict of the main character. (P17)

En todas las revisiones que abordan la construcción de los personajes, que constituyen aproximadamente un $80 \%$ del corpus analizado, los comentarios se enfocan en asuntos que podemos considerar sustantivos ya que afectan el contenido del texto y tienen un impacto directo en la experiencia de lectura a nivel macro. Así, hay comentarios, sugerencias y correcciones que tiene que ver con la relación plausible entre personajes, el balance entre descripciones psicológicas y físicas de los personajes, y la capacidad de estos para evocar emociones empáticas o antagónicas en el lector. Creemos que una de las razones detrás del gran número de revisiones en torno a este aspecto puede ser el hecho de que una de las primeras tareas del curso fue, precisamente, la construcción de un personaje convincente. Esto nos lleva a inferir que la implementación de tareas de escritura puede tener, indirectamente, un impacto en los aspectos del texto que un autor prioriza a la hora de fungir como par revisor de la producción de otro.

En lo que concierne a la construcción de audiencia, los revisores aprecian que se les ofrezcan los elementos necesarios para facilitar la comprensión estableciendo así una relación entre autor y lector en la que el primero es responsable de prever y atender las necesidades de su audiencia en términos de claridad. Los siguientes extractos ilustran esta característica:

Anyhow, I liked the way the author includes her actions in parenthesis like crying or swallowing. Those actions let me understand the situation and connect with the story better. (P16)

Something I want to highlight is how Raúl gives us background throughout all of the text so we can understand better why is this message so uncomfortable to record (...). (P18)

Este elemento de la escritura favorece la independencia del texto y da cuenta de una consciencia de audiencia que trasciende la figura habitual del profesor como interlocutor único de las producciones estudiantiles. Esto se refleja, por ejemplo, en algunas sugerencias sobre proporcionar un contexto suficiente para que otros lectores puedan entender ciertas referencias del texto, como se aprecia a continuación:

As a person that has watched Knights of the Zodiac I felt a connection with the character, and I could understand somehow why he has an introvert personality. If you want to develop this character in a deeper way, you should explain what is behind the cosmos idea, because a reader who does not know the anime would not understand your story. (P34)

Otro rasgo que aparece en los comentarios que se refieren a la construcción de audiencia es la descripción de los sentimientos y expectativas que el texto provoca, dándoles un lugar legítimo como parte de la experiencia lectora. Asimismo, los revisores valoran de manera positiva la capacidad del autor para sorprender al lector y mantenerlo enganchado:

I also liked that the text is written as a chain of situations that already happened but she would have preferred not to; their father leaving, the kicking out of their house, the mom's health problems and so on. I felt the frustration, the love. (P15) 
That "pathetic" just after the laugh made me connect immediately with the text and the character, because I could feel her desperate nervousness trying to find the right words to talk to him and not to seem like desperate or too needy. (P19)

I would like to start by saying that I didn't expect this kind of dark story coming out from Antoin. Anyways, it was a nice surprise. (P16)

Además de la construcción de audiencia, otro tema abordado por los revisores fue el cumplimiento de la tarea. Podemos afirmar que las tasks, en esta experiencia, constituyeron uno de los ejes principales del curso. Los comentarios, a continuación, así lo corroboran ya que resaltan el logro del propósito principal de la tarea propuesta:

Now, in terms of the creation of a whole scene just using noun clauses, I think this story was well done. I mean, the built up makes sense for me (...) I think Azrael doesn't say it all but with few hints and descriptions we get the full idea of what happened. Or probably what might have happened. In this sense, I think Azrael managed to accomplish the objective of this task (...) (P08)

In the exercise to detach academic and creative writing during this semester, I believe that the words used and the vocabulary are appropriate for the type of text created. (P27)

Asimismo, cuando el texto tiene carencias con respecto al objetivo de la tarea, éstas son señaladas por el revisor:
For starters, I am not really sure to what extent Raúl was able to achieve the purpose of the task through his text, because the idea of the second task was to show some features about your character through a simple or every day situation [...]. I'd say Raúl accomplished the task objective but lacked more details into his character's mind or just a description of what he thought he would do. (P20)

The writing is sober and maybe too academic in the tone. I recommend to maybe develop a little more that that you want to show as a unique sensations. Maybe develop more the extasis description instead of listing. (P31)

El tono del texto y el estilo del autor también tienen un lugar importante en las revisiones analizadas. Cabe resaltar en este apartado el papel que cumple el portafolio de cada estudiante, ya que permite una visión holística del trabajo del escritor e incluso la identificación de rasgos estilísticos como se puede apreciar en los siguientes comentarios:

"I think Azrael's texts are pretty good, full of references from other texts, movies or series". (P08).

Your humor is like your most powerful tool and if you use it to tell these parts of how the character feels because of this guy, I think it would be nice. (P19)

Otros recursos literarios que contribuyen al tono del texto son valorados positivamente $\mathrm{o}$, a falta de ellos, son sugeridos por parte del revisor; se encuentran, por ejemplo, comentarios sobre el uso efectivo de la lengua, la atmósfera creada, la inclusión de detalles sensoriales, el uso de 
metáforas, y la manera en que una secuencia de acciones es descrita:

"Can you hear me, Lord? Cause I can't hear anything" is one of the phrases that most moved me since it shows the close relationship that exists between the main character and God, that is, there is a religious and cultural atmosphere in the text. (P21)

Daniela's portray of the mess caused by a drunken man on his family's New Year party is very sincere and vivid. In my opinion, the author not only made a good job picturing the scene, but succeeded in depicting a whole relationship in just a few words. The author's use of language was very efficient, and the atmosphere was well built. (P05)

You should consider adding metaphors, comparisons... to make the text more detailed. You could look into colors meaning, for example the blue hair may refers to sadness, green to... and yellow to...(just a crazy idea). (P30)

Retomando la idea anterior, consideramos que las menciones a estos recursos literarios y estilísticos nacen de la posibilidad de una visión panorámica y progresiva sobre su obra y la de sus compañeros, posibilidad que surge gracias al uso de portafolios. A este respecto Hout (2002) identifica como uno de los fundamentos teóricos del uso del portafolio: "que los trabajos no pueden ser juzgados de manera individual, aditiva, ni fuera del contexto de otros textos en el que dichos trabajos fueron escritos" (p. 173).

El léxico también es objeto de evaluación en las revisiones analizadas. Los ejemplos a continuación muestran cómo los estudiantes articulan la escogencia de vocabulario con el campo semántico que evoca y el impacto que tiene en el tono del texto.

"The virtual way" sounds so mystic. Also, the vocabulary "match, emoji, nude" gives the modernity of the current means of relating with people. I can say I like the text, also it impresses me how fast it moves, there are I love yous and we can't really state how long it has been, but that's not a problem. (P02)

About the selection of words for this verse, I liked the mood these lines set to the verse: The Disco, Inescapable; The mind, noisy; The heart, broken. I think those lines show contrast and darkness. However, I'm still not convinced about the lights adverb, I think transcendental doesn't match the mood I just mentioned [...] I personally don't like the word pandebono there, I think it cuts the flow and rhythm of the verse, but I understand it. (P04)

Nos resulta pertinente resaltar que, en estos casos, no se trata de correcciones del vocabulario en un sentido formal, gramatical o mecánico, sino que se hacen sugerencias sobre el léxico en relación con la construcción de un tono, es decir, el léxico como recurso estilístico. Aunque sí hubo algunas correcciones de tipo formal sobre aspectos como la sintaxis, la ortografía, la concordancia verbal y la puntuación, estas no representan el foco principal de las revisiones en el corpus analizado. Este es un resultado inesperado, pues la corrección de un texto en el contexto de aprendices de lenguas, y más aún en el ámbito de formación de docentes, corresponde tradicionalmente ( $\mathrm{y}$ a veces exclusivamente) a la corrección lingüística. 
Finalmente, encontramos en el corpus apreciaciones sobre el tema escogido por el autor y la manera en que se desarrolla. Es de resaltar que en todos los casos los revisores apelan a su experiencia personal como un lugar legítimo de enunciación para justificar sus comentarios:

The first sentence hooked me. I think it's a creative way to start, and for me also an interesting way of portraying a romance. Mostly because for me a Tinder Love is not a cliché. Tinder and Tik Tok are off my radar. That's the reason I find the topic amusing and worth exploring. (P02)

First things first, I like the story, the topic is interesting, even though it has become a little cliché with movies like Fight Club and $A$ Beautiful Mind [...] there are always possibilities to use this topic to develop new perspectives. (P22)

\section{EL POSICIONAMIENTO DE LOS PARES REVISORES}

La segunda pregunta de investigación abordó el posicionamiento del revisor, a través de la cual indagamos cómo se asumen los revisores frente a sus pares autores, y cómo formulan las sugerencias y correcciones que hacen a la producción de otro. El corpus permitió la identificación de dos tipos de posicionamiento principales: comolectory, en menor medida, como autoridad. En primer lugar, el posicionamiento como lectores, presente en un $85 \%$ del corpus analizado, se hace evidente en los comentarios de unos pares revisores que desempeñan un rol de audiencia que quiere entender una historia, que quiere entretenerse con el texto, y que necesita conectarse emocionalmente con los personajes o con el tema:
It is like the reader can feel relatable with the text because we all have been there, hopeless or just wanting to do nothing in life. (P27)

I didn't feel empathy at all. Maybe you could go further into his human side. (P32)

En segundo lugar, en promedio en un $15 \%$ de los textos estudiados, aparece el posicionamiento como autoridad; este es un rol en el que el revisor se reconoce a sí mismo como alguien familiarizado con el proceso de escritura, como un autor que habla desde sus experiencias pasadas con otros textos. En este sentido, estas pocas voces de autoridad en las revisiones se caracterizaron por el uso de comandos directos por parte del revisor sobre los cambios que debe incorporar el autor:

Describe the suffering, how he feels, illustrating this would be way more interesting than the quest for a love if that's the ulterior purpose of the text. (P31)

This text is a first draft, you tried to convey many aspects of Antares. Start by telling some characteristics or aspects about Antares's wastebasket, rather than explaining his personality directly [...] (P34)

Inferimos que estos comandos directos, casi a manera de instrucciones, se desprenden de las experiencias de escritura y de las estrategias que los revisores han usado anteriormente y que, suponemos, proponen con seguridad -y autoridad- como mecanismos para el mejoramiento de la producción de sus pares. Así las cosas, podemos inferir que estos estudiantes han empezado a consolidar el yo autoral que proponen Clark e Ivanic (1997). Esto nos llevó a preguntarnos si las sugerencias se hacen de 
forma indirecta, es decir, señalando la necesidad de revisión sin proponer una solución o de forma directa, esto es, ofreciendo una solución al problema detectado (Zhao, 2018). El corpus nos ofrece evidencia de ambas instancias. En el primer caso, a pesar de no proponer una solución concreta, los extractos identificados sí incluyen explicaciones creando así la posibilidad de negociar puntos de vista:

(...) I don't understand the title and the backward title at the end. I tried to understand the "No, it is apposition", I guess it is because what was the reason to love at the beginning became the reason to break up at the end, am I right? If that's the reason ....well... I think you can do better with the title, something that shows a direct relationship with the text. (P10)

The throwing up fact, the stretch marks, are details that illustrate a lot yet the last sentence in the second paragraph didn't feel as good "It's April, and it's usually rainy" I guess was a trying to accomplish this juxtaposition of elements that we talked about, but it feels really odd and misplaced. (P14)

Las instancias en las que se señala el problema y se proponen soluciones se caracterizan por ser específicas; otras incluyen ejemplos de apartados escritos previamente por el autor como referencia para el cambio sugerido o, en algunos casos, se ofrecen alternativas para que el autor escoja lo que, a su criterio, se ajusta mejor:

[...] the only thing I would suggest is that you tell more about the man who drives your character crazy as you did in this part "remember that time when I wore that pink flowered dress in class and you smiled at me and later that day you played with my hair and accidently touched my hand?" or like the vomiting part. Your humor is like the most powerful tool and if you use it to tell these parts of how the character feels because of this guy, I think it would be nice. (P19)

In this respect, a single moment in the life of the character in which his sexuality is subtly revealed to his mother will work well to depict the universal drama of coming out. Details will lead the author to the whole picture. Maybe focusing on the doll episode or the pink dress event, how the mother perceived what her son was, will work. (P17)

Otra estrategia usada cuando se señalan problemas es la de hacer preguntas que interpelan directamente al autor y que funcionan como dispositivos para la reflexión sobre las decisiones tomadas:

I would also like to see a deeper development on the tension between the mother's religion and acceptance of his son's sexuality. The character of the mother reveals a duality: the precepts of her religion and her unconditional love for her son. How strong is that inner conflict for her? I think giving a deeper view of the mother's conflict would intensify the conflict of the main character. (P17)

Con respecto a los patrones de interacción encontramos que la mitigación, entendida como unaestrategiaque "modificalafuerzailocucionaria y por tanto el status epistémico o deóntico de las expresiones" y que usa como dispositivos "Ios diminutivos o partículas argumentativas, tag questions, subjuntivo, vacilaciones, expresiones vagas, etc. (Wodak, 2016, p. 6) precede casi siempre a la formulación de una sugerencia o al 
señalamiento de un problema. Este fenómeno coincide con los postulados de Goffman en relación con la tendencia de los miembros de un grupo "a mantener un estándar de consideración para salvar los sentimientos y la cara de los demás de manera voluntaria y espontánea dada la identificación emocional con los otros" (Goffman, 1967, p. 10). Una estrategia de mitigación recurrente en el corpus corresponde a una suerte de concesión: primero se ofrece un comentario positivo o se elogia un aspecto del texto, y luego se introduce una crítica, una corrección o un aspecto a mejorar. Los extractos a continuación ejemplifican cómo los revisores expresan honestamente sus opiniones sin por ello sacrificar el tacto y delicadeza:

Finally Free Verse, I liked it a lot; the main words of the text build the story, but when you get to the long sentence it's like the weak part of the text, I don't know, I think it could be stronger as the beginning and the ending. (P07)

I loved the way you mixed mathematical terms in your text. However, there isn't clear the role that played the horse in Hesitation. (P39)

En general, la concesión como estrategia de mitigación aparece a lo largo del corpus en combinación con el uso de los modales would, should, y could, y en consonancia con un registro muy familiar a la hora de escribir las críticas por parte de los revisores; se trata de una suerte de transliteración del registro oral a la escritura, que interpretamos como una manera de mantener cercanía con el autor del texto, y hacerle críticas y correcciones desde una interacción de confianza y camaradería que guarde la cara de ambos, en términos de Goffman (1967). Finalmente, junto a este recurso de diálogo oral llevado a la escritura, encontramos una cantidad considerable de emoticones ${ }^{4}$ que acompañan

$4 \quad$ Aparecen en el corpus emoticones que representan una cara feliz (:) ), una cara tierna (:3) o una cara aver$\operatorname{gonzada}(>.<)$. las críticas y sugerencias; creemos que, ante la falta de prosodia, los revisores incluyeron estos emoticones como una forma de suavizar sus comentarios y transmitir su actitud positiva en las críticas y sugerencias ofrecidas al autor.

\section{CONCLUSIONES}

Una de las dificultades más frecuentemente identificadas en las experiencias de uso de la revisión de pares como estrategia metodológica para la enseñanza de la escritura está relacionada con la superficialidad de los comentarios, que se concentran en su mayoría en aspectos formales del texto. Las revisiones aquí analizadas muestran lo contrario, ya que se enfocan en aspectos globales tales como la construcción de personajes, la construcción de audiencia, el tema, el estilo y el cumplimiento de la tarea y representan una retroalimentación significativa que ofrece al autor elementos para la reflexión y la reescritura.

Abordar de manera explícita el concepto de revisión de pares así como destinar un tiempo importante del trabajo en el aula para la discusión de la metodología y del rol que se esperaba que asumieran los estudiantes como revisores fueron acciones que favorecieron, en la experiencia aquí reportada, la implementación de esta práctica de manera exitosa.

La revisión de pares ofrece elementos para la promoción de la cooperación y la construcción colectiva de conocimiento siempre y cuando se cree un ambiente de confianza y camaradería donde todos los participantes se sientan seguros para exponerse a la mirada del otro y para reconocerse mutuamente como interlocutores legítimos que pueden aportar no solo al mejoramiento de un texto en particular sino al desarrollo de las capacidades del escritor. En este estudio de caso, el posicionamiento de los revisores reveló una actitud respetuosa del 
trabajo de sus compañeros, predominando una visión de sí mismos como audiencia que hace pedidos específicos en pro de mejorar el texto, y facilitar así la lectura a otros lectores potenciales. Tal actitud respetuosa está presente incluso en aquellos casos en los que se percibe el desarrollo del yo autoral; aunque aún en ciernes, este último permitió a los pares revisores sugerir cambios de manera contundente desde su conocimiento y experiencias pasadas, sin comprometer su cara ni la de sus pares autores. Las estrategias de mitigación encontradas en el corpus son garantes de esto último.

La revisión de pares exige una visión del texto como material abierto y susceptible de ser mejorado, lo cual supone superar la idea de la escritura como algo terminado, que se cristaliza una vez se termina la primera versión. En este sentido, las revisiones de los pares se convierten en "mapas de ruta que resaltan el estatus presente del texto y su futura trayectoria" (Anson \& Anson, 2017, p. 13) y permiten a los estudiantes trascender la tradición de corrección de errores, tan característica en los procesos de enseñanza de escritura en lengua extranjera.

En el corpus analizado, los revisores logran un balance entre elogios y crítica que favorece el intercambio amable y honesto de experiencias de lectura y escritura y motiva a los escritores. De otra parte, al ocupar alternativamente el lugar de escritor y revisor, los estudiantes logran analizar los textos de una manera profunda, que indaga en las razones por las que los escritores "toman ciertas decisiones en términos de contenido, organización y estilo" (Cahill, 2002, p. 303) y se hacen conscientes de "qué es lo que están tratando de crear y qué tan bien sus borradores responden a los objetivos lingüísticos y retóricos que se han trazado".(Huot, 2002, p. 170). En este sentido, el uso de las tareas (tasks) resultó aliado valioso para el curso de escritura creativa porque permitió, por un lado, exponer a los estudiantes a situaciones auténticas de escritura para una audiencia real, y por otro, les permitió actuar como revisores cuya herramientas se fueron puliendo con las características de cada tarea.

La implementación de la revisión de pares como estrategia pedagógica implica una transformación de los roles de los estudiantes y por consiguiente, del rol del profesor. El maestro en este contexto ha de estar dispuesto a ceder el control pero esto no significa que deje de guiar el proceso. Su papel como organizador de las dinámicas de lectura y escritura, su intervención cuando sea necesario para garantizar que la retroalimentación sea sincera pero no por ello destructiva y su responsabilidad como diseñador de insumos para la comprensión y realización de esta práctica son esenciales. Asimismo, con respecto al rol del profesor, vale la pena reflexionar sobre el importante papel que juega el tipo de retroalimentación y correcciones que hacemos de los textos de nuestros estudiantes; estas tienen, en gran medida, el poder de modelar y determinar el posicionamiento de los revisores y el foco de las revisiones que hacen sobre la producción de otros. Una mirada sistemática a las revisiones de pares hechas por estudiantes pueden revelar aspectos ocultos de cómo asumimos los docentes esta tarea, así como la inherente responsabilidad de evaluar $y$ muchas veces calificar la producción escrita de los estudiantes.

\section{REFERENCIAS BIBLIOGRÁFICAS}

Akhtar, F. (2015). Reclaiming the "Wounded Storyteller": The Use of Peer Feedback as a Pedagogical Tool in Creative Life Writing. Writing in Practice, 1, 24-35

Anson, I. G., \& Anson, C. M. (2017). Assessing peer and instructor response to writing: A corpus analysis from an expert survey. Assessing Writing, 33, 12-24. https:// doi.org/10.1016/j.asw.2017.03.001 
Bijami, M., Kashef, S. H., \& Nejad, M. S. (2013). Peer Feedback in Learning English Writing: Advantages and Disadvantages. Journal of Studies in Education, 3(4), 91.

Braun, V.\& Clarke, V. (2006). Using thematic analysis in psychology. Qualitative Research in Psychology, 3(2), 77-101.

Cahill, L. (2002). Reflection on Peer Review Practices. En Roen, D. et al. Strategies for Teaching First-Year Composition. (pp. 301-306) National Council of Teachers.

Colombo, L., \& Álvarez, G. (2021). Iniciativas didácticas basadas en la revisión entre pares y orientadas a la enseñanzaaprendizaje de la escritura de tesis a nivel de posgrado. Educación y Humanismo, 23(40).

269 . Chois-Lenis, P. M., López-Higuera, A., PradoMosquera, D. M., Cajas-paz, E. Y., \& Casas-Bustillo, A. C. (2017). Percepciones sobre la tutoría entre pares en escritura académica. Magis, Revista Internacional De Investigación En Educación, 9(19), 165-184.

Clark, R. \& Ivanic, R. (1997). The politics of writing. Routledge.

Gérvas, J., \& Pérez Fernández, M. (2001). La revisión por pares en las revistas científicas. Atención Primaria, 27(6), 432-439.

Harris, M. (1992). Collaboration Is Not Collaboration Is Not Collaboration: Writing Center Tutorials vs. PeerResponse Groups. College Composition and Communication, 43(3), 369-383.
Hyland, K., \& Hyland, F. (2006). Feedback on second language students' writing. Language Teaching, 39(2), 83-101.

Huot, B. (2002) Toward a new discourse of assessment for the college writing classroom. College English 65(2), 163180.

López, K. S. (2012). La revisión entre pares de tareas de escritura como herramienta de una didáctica metacognitiva en el aula de lengua. Lenguaje, 40(2), 351-381.

Nelson, G. \& Carson, J.G. (1998). ESL students' perceptions of effectiveness in peer response groups. Journal of Second Language Writing, 7(2), 113-131.

Norton, S. (2013). Betwixt and Between: Creative Writing and Scholarly Expectations. New Writing, 10(1), 68-76.

Paton, F. (2002). Approaches to Productive Peer Review. En Roen, D. et al. Strategies for Teaching First-Year Composition. (pp. 290-301) National Council of Teachers.

Salinas, A. M. (2020). Writing development through materials that focus on peer feedback in an EFL context. ENLETAWA Journal, 13(1), 20-56.

Sanabria M, M. (2015). Student-teachers as editors and writers: a pedagogical design to examine peer edition through formative assessment. Enletawa Journal, 1, (8), 83-104.

Schneider, B., \& Andre, J. (2007). Developing authority in student writing through written peer critique in the disciplines. The Writing Instructor 2007. 
Sengupta, S. (1998). Peer evaluation: 'I am not the teacher'. ELT Journal, 52(1), 19-28.

Tsui, A. B. M., \& Ng, M. (2000). Do secondary L2 writers benefit from peer comments? Journal of Second Language Writing, 9 , 147-170.

Vaismoradi, M., Turunen, H., \& Bondas, T. (2013). Content analysis and thematic analysis: Implications for conducting a qualitative descriptive study. Nursing \& health sciences, 15(3), 398-405.

Vargas Franco, A. (2014). Revisión entre iguales y escritura académica en la universidad: la perspectiva del estudiante. Folios, 39 , 13-29.

Wodak, R. (2016). "We Have the Character of an Island Nation". A Discourse-Historical Analysis of David Cameron's "Bloomberg Speech" on the European Union. SSRN Electronic Journal. Published. https:// doi.org/10.2139/ssrn.2870570

Zhang, S. (1995). Re-examining the affective advantages of peer feedback in the ESL writing class. Journal of Second Language Writing, 4(3), 209-222.

Zhao, H. (2018). New insights into the process of peer review for EFL writing: A processoriented socio-cultural perspective. Learning and Instruction, 58, 263-273. 